\title{
Safety checks and hit probability computation in numerical trajectory calculation using error propagation technology
}

\author{
A. Kuhrt \& H. Rothe \\ University of the Federal Armed Forces, Hamburg, Germany
}

\begin{abstract}
In fire control systems using numerical trajectory integration, safety checks and hit probability information still is based on empirical parameters. The need for a modern and precise security testing on a defined level of confidence and tailored hit probability computation were the motivation to develop the technology outlined in this paper. The methods are based on trajectory calculation using a modified point mass model that considers drag, lift, Magnus and Coriolis forces, gravity and all atmospheric influences. The Gaussian law of the propagation of the stochastic error within a system of dependent and independent variables was used to generate a vector of standard deviations for all error afflicted parameters every integration time step. This approach leads to a flightpath dataset of the projectile, not only giving information about position and velocity, but also about their standard deviations. The check for a minimum height over ground or distance to no-fly zones is very easy and can be performed with a changing level of confidence throughout the trajectory. Hit probability calculation is done automatically and considers all current data that has influence on the projectile's trajectory.
\end{abstract}

Keywords: error propagation, safety checks, numerical integration, crest clearance, hit probability, multivariate statistics.

\section{Introduction}

In fire control systems using numerical trajectory integration, safety checks and hit probability information still is based on empirical parameters. Even modern systems using time consuming routines on calculating numerical derivatives for a trajectory perturbation in order to gain hit probability information. The need for a 
precise security testing on a defined level of confidence and tailored hit probability computation were the motivation to develop the technology outlined in this paper. The methods are based on trajectory calculation using a modified point mass model that considers drag, lift, Magnus and Coriolis forces, gravity and all atmospheric influences.

\section{Fire control software}

Modern fire control software mostly is strongly unitized to cover a wider range of different weapon systems or ammunition types. This leads to a data-handling that has to be taken into consideration. Trajectory calculation has to meet the demands on both, computation time and accuracy. That influences the numerical method and the physical model used.

\subsection{Trajectory calculation}

The trajectory calculation used in modern Fire Control Software is mostly based on a set of differential equations (DEQ) incorporating a modified point mass model $[1,2]$. These differential equations are solved by a numerical integration scheme to generate a flightpath containing the important information such as position, velocity and angle for each time step from leaving the muzzle to the impact.

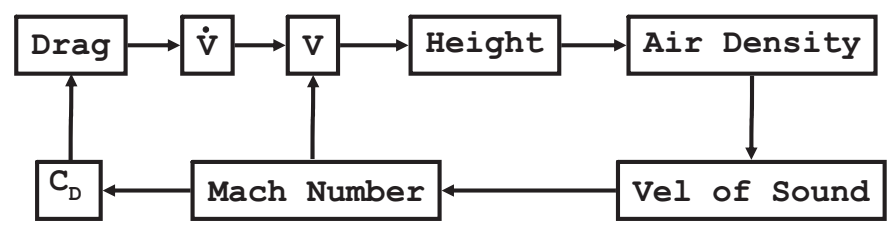

Figure 1: Example for the Dependencies of integration parameter Drag.

Common integration schemes used are explicit or implicit single-step methods, such as Heun or Runge-Kutta. Due to the good conditioned DEQs faster schemes without error-estimation were preferred to keep computation time short. The algorithms described below are developed for an Euler scheme, but were also successfully tested on a Heun scheme.

During numerical integration of the trajectory parameters change, depending on current projectile position and velocity. Atmospheric data gained from measurements or meteorological prognosis depends strongly on the height over ground. Ballistic coefficients may depend on the Mach number, which on its part depends on the actual speed of sound and the projectile velocity. There are many parameters in the DEQs of motion that depend on the results of the last performed integration step or that are linked to external data sets (e.g. fig. 1). 


\subsection{Requirements for safety checks}

In modern fire control software an important part is security. Therefore, a wide range of tests were provided to check the interaction of a trajectory with other objects or zones. These zones can vary between traditional checks against trajectory-ground interaction and checks against violation of temporary no-fly zones. Today's checks are mostly performed by perturbation mathematics and their application on the trajectory [3]:

Adding a perturbation on one initial condition and calculating the difference of the hit point allows to compute the numerical partial derivative with respect to the initial condition perturbed. The linear combination of these partial derivatives multiplied with their variances leads to the absolute error probability usually given as a CEP. The mathematical background is the Gaussian law of the propagation of the stochastic error [4]:

$$
\sigma_{y}^{2}=\sum_{i=1}^{N}\left(\frac{\partial f}{\partial x_{i}}\right)^{2} \sigma_{i}^{2}=\left(\frac{\partial f}{\partial x_{1}}\right)^{2} \sigma_{1}^{2}+\left(\frac{\partial f}{\partial x_{2}}\right)^{2} \sigma_{2}^{2}+\left(\frac{\partial f}{\partial x_{3}}\right)^{2} \sigma_{3}^{2}+\cdots
$$

This approach is also suitable for crest clearance checks:

Two perturbed trajectories were calculated which enclosure the original trajectory. One of this new trajectories - the lower one - is used for checking against projectileground-interaction. The higher one is obsolete.

Another approach is to rise the ground to a safety level needed and then check the original trajectory against projectile-ground-interaction using the raised ground level.

Both algorithms can only handle defined and fixed error budgets for a limited number of parameters because every parameter to be perturbed causes an additional complete trajectory calculation. These parameters are limited to initial conditions of the numerical integration and no dependencies were taken into consideration.

\section{A measurement approach}

In measurement theory basically all measurands are error afflicted. These errors are quantified by two characteristic values: mean value of the sample $(\bar{x})$ and standard deviation of the sample $\left(s_{x}\right)$. All error afflicted measurands are assumed Gaussian distributed.

Looking at the parameters affecting a trajectory integration, three main categories can be differentiated:

1. Parameters directly affecting the initial conditions, such as gun position, gun height, muzzle velocity, azimuth and elevation.

2. Parameters indirectly affecting the initial conditions, like propellant temperature and barrel abrasion.

3. Parameters directly affecting the projectile in flight containing all atmospheric properties depending on height (temperature, air density, air 
pressure, wind, speed of sound) and all projectile depending coefficients (drag, lift, Magnus and Coriolis forces, spin, yaw).

From a measurement point of view, all these parameters are error afflicted and therefore, every parameter can be quantified by its mean and standard deviation. The aim is to get a statement for the certainty - or better uncertainty - of the projectile's position and velocity. The approach is to propagate the error of the position and the velocity vector not by perturbing the initial conditions, but by recalculating the standard deviation of position and velocity vector every integration time step.

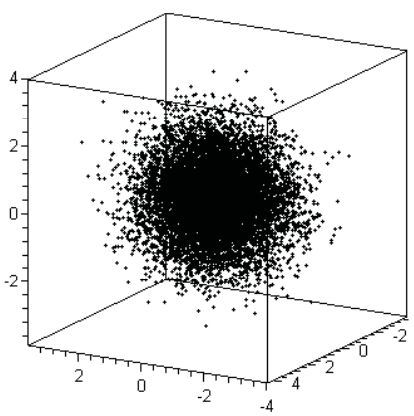

Figure 2: Resulting fuzzy position of a projectile.

The main advance is that a data set of projectile's uncertainty for each point of the trajectory calculated is produced. Changing standard deviation of error afflicted parameters during the trajectory is possible and therefore assures a good flexibility. This computation is extraordinary fast compared to an equivalent perturbation approach.

\section{Error propagation}

In general, the Gaussian Law (eqn. (1)) covers most of normal measurement cases. But because modern fire control softwares integration methods and object handling splits up the computation of position and velocity vector into subroutines, a more general definition of the error propagation has to be used. The variances and their depending variables to be added at the end of the calculation cannot be assumed to be independent anymore. To consider these dependencies the general law for error propagation has to be used [5]: Let $f\left(x_{1}, x_{2}, \ldots, x_{n}\right)$ be a function which depends on $n$ variables $x_{1}, x_{2}, \ldots, x_{n}$. The uncertainty of each variable is given by $\sigma_{x_{j}}$ multiplied with the quantile for a level of confidence of $\alpha$ (remark, that in military applications times of PE is the common quantile): If the variables are correlated, 
the covariance between variable pairs, $C_{i, k}:=\operatorname{cov}\left(x_{i}, x_{k}\right)$, enters eqn. (1) with a double sum over all pairs $(i, k)$ :

$$
\sigma_{f}=\sqrt{\sum_{i=1}^{n} \sum_{k=1}^{n}\left(\frac{\partial f}{\partial x_{i}} \frac{\partial f}{\partial x_{k}} C_{i, k}\right)},
$$

where $\frac{\partial f}{\partial x_{j}}$ designates the partial derivative of $f$ for the $j$-th variable and $C_{i, i}=$ $\operatorname{var}\left(x_{i}\right)=\sigma_{x_{i}}^{2}$. After calculating $\sigma_{f}$, the value of the function with its uncertainty is:

$$
f \pm u_{\alpha / 2} \sigma_{f}
$$

The estimation of the correlation matrix is discussed in section 5. Within numerical integration schemes error propagation does not follow this law.

\subsection{Error propagation in Euler schemes}

In Euler schemes every discretized equation can be written as:

$$
x_{i}=x_{i-1}+g_{i} \Delta t_{i},
$$

with $\Delta t_{i}$ as the not error afflicted, but varying integration increment, $g_{i}$ as a function, varying and error afflicted, that incorporates the discretized DEQ within increment $i$.

Analysis following the method described in section 5 shows that the correlation between $x_{i-1}$ and $g_{i}$ is negligible. This would enforce a Gaussian error propagation approach. Trying this one will end up with wrong results, because this approach considers only the increment $\Delta t_{i} \in\left[0, \Delta t_{i}\right]$, but neglects the actual position within the integration process, which would be $t \in[0, t]$. Instead an error propagation similar to the integration scheme used is postulated:

$$
\sigma_{x_{i}}=\sigma_{x_{i-1}}+\sqrt{\sigma_{x_{0}}^{2}+t^{2} \sigma_{g_{i}}^{2}}-\sqrt{\sigma_{x_{0}}^{2}+\left(t-\Delta t_{i}\right)^{2} \sigma_{g_{i}}^{2}}
$$

In fact, the equation shown is a steady continuation of the existing error with a gradient with respect to the actual integration increment.

The main advantage is to consider the whole integration time $t$ and the initial error. The following figures show the differences of a Gaussian and this approach for the error in velocity and range of a constantly slowed down vehicle from $30 \mathrm{~m} / \mathrm{s}$ with an error in $v_{0}$ and in the friction co-efficient: 

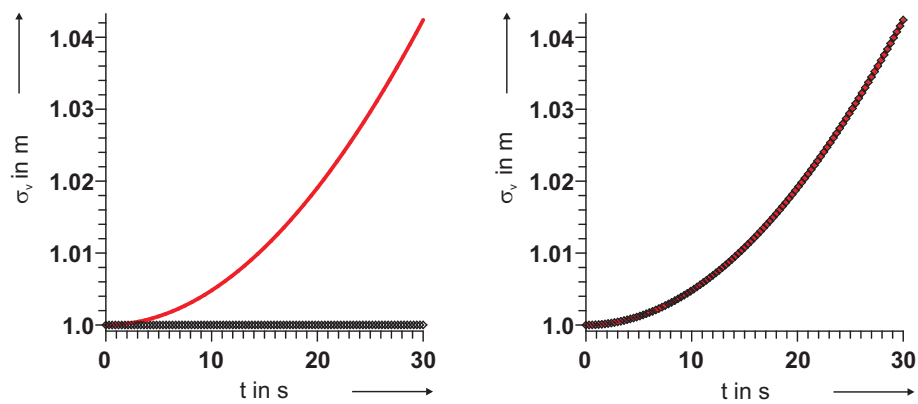

Figure 3: Resulting standard deviation of $v$ of analytical solution (grey) and of Euler scheme (black); Left: Gaussian law Right: New approach.
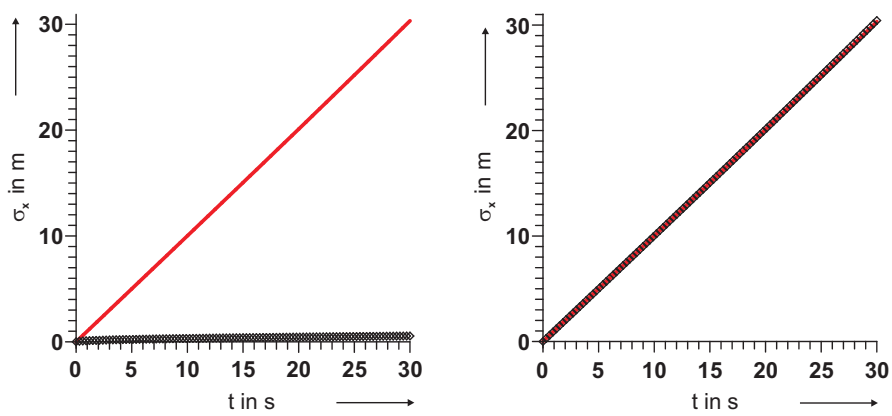

Figure 4: Resulting standard deviation of $x$ of analytical solution (grey) and of Euler scheme (black); Left: Gaussian law Right: New approach.

\section{Estimation of correlation coefficients}

For the calculation of the error propagation, the covariances between pairs of parameters must be known. The general definition of the covariance is:

$$
\begin{aligned}
\operatorname{cov}\left(X_{1}, X_{2}\right) & =E\left(X_{1} \cdot X_{2}\right)-E\left(X_{1}\right) \cdot E\left(X_{2}\right) \\
& =\rho \cdot \sigma_{1}^{2} \cdot \sigma_{2}^{2} \\
& =\int_{\infty}^{-\infty} \int_{\infty}^{-\infty} x_{1} \cdot x_{2} f\left(x_{1}, x_{2}\right) \mathrm{d} x_{1} \mathrm{~d} x_{2}-E\left(X_{1}\right) \cdot E\left(X_{2}\right)
\end{aligned}
$$

with $X_{1}, X_{2}$ as variates, $E(\ldots)$ as the expectant and $\rho$ as the the Pearson product-moment correlation coefficient (PMCC). Mind that the probability density function (PDF) $f\left(x_{1}, x_{2}\right)$ is a bivariate Gaussian distribution with the Gaussian distributions of each error afflicted parameter as marginal distributions. The difficulty to estimate the covariance of two parameters is, that the transformation of bi-variate distribution to the marginal distributions is irreversible. The bi-variate 
Gaussian PDF is defined by 5 parameters: $\mu_{x_{1}}, \mu_{x_{2}}, \sigma_{x_{1}}, \sigma_{x_{2}}, \rho$.

$$
f\left(x_{1}, x_{2}\right)=\frac{\mathbf{e}^{-\frac{1}{1-\rho^{2}}\left[\frac{\left(x_{1}-\mu_{x_{1}}\right)^{2}}{2 \sigma_{x_{1}}^{2}}-\rho \frac{\left(x_{1}-\mu_{x_{1}}\right)\left(x_{2}-\mu_{x_{2}}\right)}{\sigma_{x_{1}} \sigma_{x_{2}}}+\frac{\left(x_{2}-\mu_{x_{2}}\right)^{2}}{2 \sigma_{x_{2}}^{2}}\right]}}{2 \pi \sigma_{x_{1}} \sigma_{x_{1}} \sqrt{1-\rho^{2}}}
$$

The extraction of the marginal distributions is easy and like:

$$
f_{x_{1}}\left(x_{1}\right)=\frac{1}{\sqrt{2 \pi} \sigma_{x_{1}}} \mathbf{e}^{-\frac{\left(x_{1}-\mu_{x_{1}}\right)^{2}}{2 \sigma_{x_{1}}^{2}}}, \quad f_{x_{2}}\left(x_{2}\right)=\frac{1}{\sqrt{2 \pi} \sigma_{x_{2}}} \mathbf{e}^{-\frac{\left(x_{2}-\mu_{x_{2}}\right)^{2}}{2 \sigma_{x_{2}}^{2}}},
$$

but the reconstruction of the bivariate PDF is impossible without the defining parameter $\rho$ [6]. The approach is to determine this PMCCs using a pseudo empiric approach.

\subsection{Pseudo-empiric correlation matrix}

Although the error afflicted parameters used in fire control software are populations, the method to estimate the PMCC is derived from a method developed for samples. The way to determine an empiric correlation matrix, as described in [7], is:

$j$ variates are determined in $i$ samples. The values of the variates are standardized by subtracting the arithmetic mean value of the variate and dividing it by the standard deviation of the variate throughout the samples.

$$
z_{i j}=\frac{x_{i j}-\bar{x}_{j}}{s_{j}}
$$

with

$z_{i j}$ : standardized value of the variate $j$ in sample $i$.

$\bar{x}_{j}$ : arithmetic mean of the variate $j: \bar{x}_{j}=\frac{1}{n} \sum_{i=1}^{n} x_{i j}$.

$s_{j}$ : empiric standard deviation of the variate $j$ :

$$
s_{j}=\sqrt{\frac{1}{n-1} \sum_{i=1}^{n}\left(x_{i j}-\bar{x}_{j}^{2}\right)} .
$$

This standardization results in a $n \cdot m$ matrix $Z$ :

$$
Z=\left(\begin{array}{cccc}
z_{11} & z_{12} & \cdots & z_{1 m} \\
z_{21} & z_{22} & \cdots & z_{2 m} \\
\vdots & & & \\
z_{n 1} & z_{n 2} & \cdots & z_{n m}
\end{array}\right)
$$

The empiric correlation matrix $R$ is then:

$$
R=\frac{1}{n-1} Z^{T} Z
$$

The expectation value of the empiric correlation matrix $R$ is the correlation matrix of the population $P=E(R)$. 
The approach is to generate samples out of the error afflicted equation using the conditional equations of the error afflicted parameters.

1. Each error afflicted parameter is defined as a variate.

2. Each error afflicted parameter appearing in a conditional equation generates a sample.

3. A sample is generated by perturbing the parameter defined in 2 . within all conditional equations by its standard deviation $\sigma$.

4. The results are standardized using the means and standard deviations of the variate populations.

The resulting matrix consists of $n \cdot m$ samples $z, n$ variates $f_{x_{n}}$, depending on $m$ parameters $a$ :

$$
Z=\left(\begin{array}{ccc}
z_{11}=f_{x_{1}}\left(a_{1}+\sigma_{a_{1}}, \cdots, a_{m}\right) & \ldots & z_{1 m}=f_{x_{1}}\left(a_{1}, \cdots, a_{m}+\sigma_{a_{m}}\right) \\
\vdots & & \vdots \\
z_{n 1}=f_{x_{n}}\left(a_{1}+\sigma_{a_{1}}, \cdots, a_{m}\right) & \ldots & z_{n m}=f_{x_{n}}\left(a_{1}, \cdots, a_{m}+\sigma_{a_{m}}\right)
\end{array}\right)
$$

The sum of each column is 1. Each sample adds the part of the PMCC generated by the perturbed parameter. Perturbed parameters adding parts to more than one variate cause correlations. Calculating $R$ using eqn. (13) provides an equation for every PMCC. The division by $n-1$ drops out because the use of standard deviation generates theoretical infinite sized samples and the resulting matrix is in fact the correlation matrix of the population $P$. This approach was verified and successfully tested using Monte-Carlo simulations [8].

\section{Example}

As an example for safety testing of trajectories an artillery shell was chosen. It is fired with a quadrant elevation (QE) of $800^{-}$. The trajectory was computed using a predictor-corrector integration scheme for a modified point mass model (MPMM). Only a few parameters were considered error afflicted, such as the drag coefficient, projectile mass, QE and muzzle velocity. Using the error propagation technology, outlined here, a plot can be generated that shows the position of the projectile at any point of the trajectory on a defined level of confidence. Figure 5 shows areas of positional probability on a level of confidence of $10 \mathrm{PE}$ which is equivalent to $99.93 \%$. In the first plot elipsoids for $10 \mathrm{PE}$ for range and height are given. The second plot shows elipsoids for range and deflection.

For safety tests the most important information is the distance between projectile and ground measured in times of PE. Figure 6 shows on the left the trajectory and the corresponding ground profile. On the right the times of PE are plotted together with the changing safety level regarding the projectile's traveling over own (20 PE) or foe (10 PE) ground or while approaching the target (0 PE). 

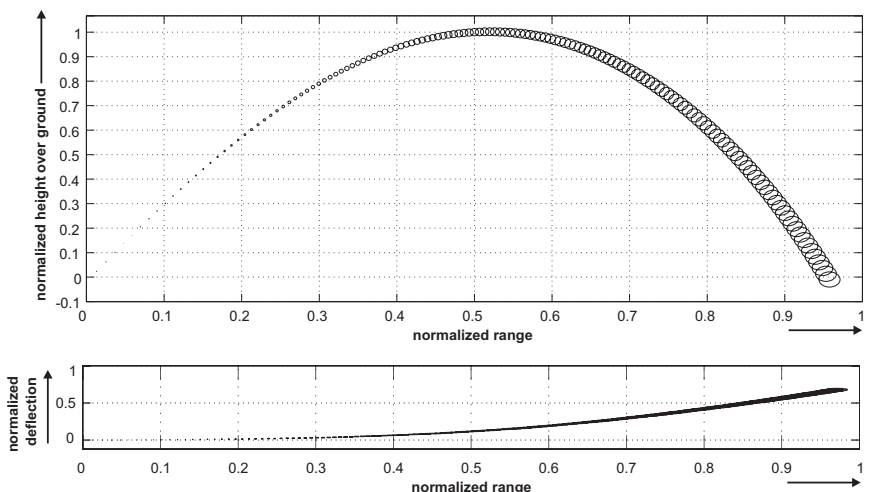

Figure 5: Probable projectile position on a C.L. of 50 PE.
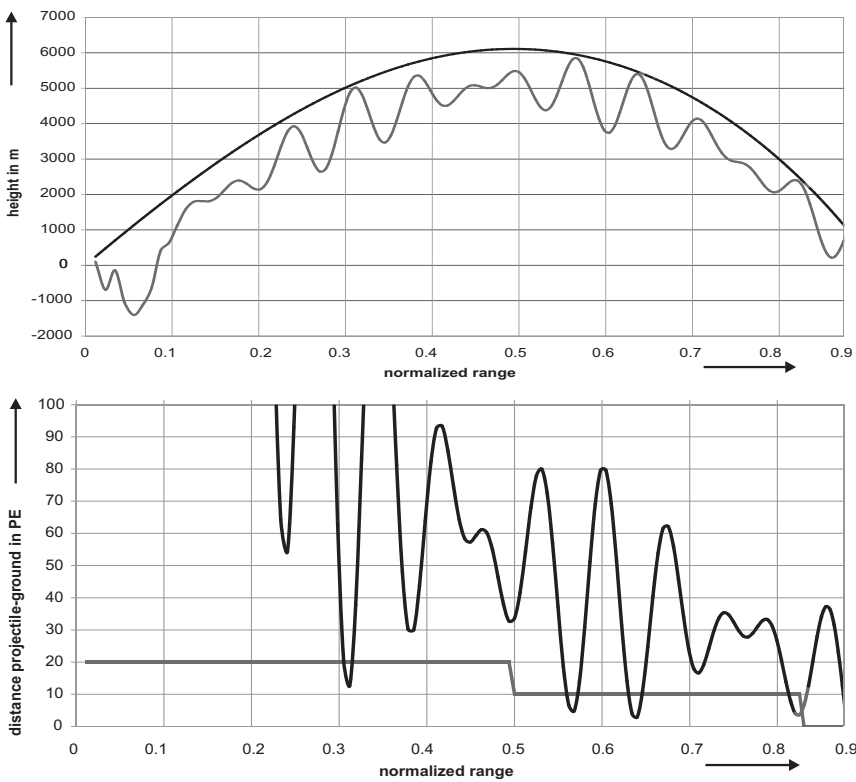

Figure 6: Safety in Crest checking in times of PE.

\section{Conclusions}

The technology of error propagation outlined in this paper is a new approach in calculating PEs. The current technology of overall numerical derivation with respect to some parameters is replaced by an analytical derivation with respect to all parameters and performed within each integration time step. This allows the change of standard deviation throughout the flightpath of the projectile. 
A new method for the propagation of error afflicted parameters within first and second order numerical integration schemes has been developed and successfully tested. The correlation between parameters assumed error afflicted has been determined using a new quasi empiric approach. The resulting PMCC matrices vary throughout the flightpath and can be recalculated every integration time step.

In result, flightpath data with information about error probability for position and velocity for each integration time step is given. Calculation of the probable error at the target is done automatically and with good accuracy even for deflection, that was determined empirical before. Safety testing against trajectory - ground interaction can be performed on a high level of flexibility by changing safety quantiles during the flight. Checks against no-fly zones now can be performed by a fraction of the effort formerly necessary.

The usage of the technology outlined is not restricted to trajectory calculation, but offers the opportunities for many other branches using time critical numerical initial value solvers.

\section{References}

[1] NATO, STANAG 4355 - The Modified Point Mass and five Degrees of Freedom Trajectory Models. 4th edition, 2006.

[2] McCoy, R.L., Modern Exterior Ballistics. Schiffer Military History: Atglen, 1999.

[3] Molitz, R., Hellmuth; Strobel, Äußere Ballistik. Springer: Berlin, 1963.

[4] Adunka, F., Meßunsicherheiten - Theorie und Praxis. Vulkan-Verlag: Essen, 2000.

[5] Irle, A., Wahrscheinlichkeitstheorie und Statistik. Teubner: Stuttgart, Leipzig, Wiesbaden, 2001.

[6] Cook, S., R. Dennis; Weisberg, Applied Regression including Computing and Graphics. Wiley: New York, 1999.

[7] Eckey, R.R.M., Hans-Friedrich; Kosfeld, Mulitvariate Statistik: Grundlagen Methoden - Beispiele. Gabler: Wiesbaden, 1st edition, 2002.

[8] Kuhrt, A., Crest Clearance und ACA Check Technologie für numerische Flugbahnberechnungen - Theoretische Grundlagen. Research Report: Hamburg, 2007. 